\title{
Review of: "Global spread of Salmonella Enteritidis via centralized sourcing and international trade of poultry breeding stocks"
}

\author{
Song $\mathrm{Gao}^{1}$ \\ 1 Yangzhou University
}

Potential competing interests: The author(s) declared that no potential competing interests exist.

By integrating over 30,000 Salmonella Enteritidis genomes from 98 countries during 1949-2020 and international trade of live poultry from the 1980s to the late 2010s, authors present multifaceted evidence that converges on a high likelihood, global scale, and extended protraction of Salmonella Enteritidis dissemination via centralized sourcing and international trade of breeding stocks. In this paper, authors also identified concordant patterns of international trade of breeding stocks and quantitatively established a driving role of the trade in the geographic dispersal of Salmonella Enteritidis. The data employed in this study are massive, and the conclusions drawn by authors are credible. To further explore the interests and/or information of this this study, there are some suggestions as following:

1. From the global SNP phylogeny, authors identified three major lineages of poultry isolates: an international lineage represented by isolates from all inhabited continents (Global); another international lineage dominated by European and US isolates (Atlantic); and an overwhelmingly US lineage (US). Are there specific molecular markers or SNPs to distinguish these three lineages besides their geographic origin? It is important for a given individual Salmonella Enteritidis isolate to be classified into one of the three lineages and to be traced its origin. The authors might further detail the criteria for the lineage classification.

2. Within poultry, the mixture of isolates from chicken and eggs suggests indistinguishable Salmonella Enteritidis populations circulating in both broilers and layers. Based on this study, are there any differences of susceptibility to Salmonella Enteritidis infection between broilers and layers? And are there any differences in Salmonella Enteritidis isolation rates between countries overwhelmingly imported broilers and those tended to import layers?

3. As the biggest importer, China mainland had imported live poultry originated from Europe and the US at a \$754 million level between 1962 and 2019. According to authors' opinion, which is the overwhelming lineage circulating in China among Global, Atlantic and US lineages of Salmonella Enteritidis defined by this study? And what are the potential causes to result in the superior lineage? It might be helpful for importers to understand the origin of Salmonella Enteritidis upon international trade.

4. In many developing countries(including China), additional S. enterica serotypes, especially serotypes Pullorum and Gallinarum are common in poultry flocks. When Salmonella Enteritidis introduced, are 
there potential interferences between isolates belonging to these different serotypes? If yes, could the situation have an effect on Salmonella Enteritidis isolation rate and further its infection prevalence in these countries? Authors are encouraged to discuss about this concern. 